\title{
THE DYNAMICS OF SOLAR CORONAL MAGNETIC FIELDS
}

\author{
B. C. Low \\ High Altitude Observatory \\ National Center for Atmospheric Research \\ Boulder, Colorado, USA
}

The solar corona is a hot $\left(10^{6} \mathrm{~K}\right)$ highly ionized plasma structured by its magnetic field into open regions where the solar wind escapes and closed regions where relatively dense plasma is trapped in near static equilibrium. Observed in Thomson-scattered light at times of solar eclipse or by artificial occultation using a coronagraph, these closed regions show up conspicuously as helmet-shaped bright structures. The large scale corona evolves in time in response to the solar dynamo that continually injects new magnetic flux into the corona with the eventual reversal of the global magnetic polarity at the end of each half cycle of eleven years. It was discovered in the 1970 s using spaceborne coronagraphs that in addition to its long-term evolution, the corona also undergoes dynamical reconfiguration with ejection of mass of the order of $10^{15} \mathrm{~g}$ into interplanetary space (MacQueen 1980). These time dependent phenomena take place once every few days at solar activity minimum and as often three times a day at solar activity maximum. Since the 1970s, coronal mass ejections have been studied at the High Altitude Observatory by the use of the coronagraph on the NASA Solar Maximum Mission Satellite and groundbased instruments at Hawaii. This brief review presents three points of interest in the coronal mass ejection as a hydromagnetic process, emphasizing the unique opportunity offered by the corona to study hydromagnetic phenomena by direct observation (Hundhausen 1987, Kahler 1987, Low 1986).

Coronal mass ejections are observed to have speeds falling in a broad range from 10 to $10^{3} \mathrm{~km} \mathrm{~s}^{-1}$ with a median of about $350 \mathrm{~km} \mathrm{~s}^{-1}$. This median speed is greater than the coronal sound speed $\left(160 \mathrm{~km} \mathrm{~s}^{-1}\right)$ but less than the typical Alfven speed $\left(>600 \mathrm{~km} \mathrm{~s}^{-1}\right)$ and the gravitational escape speed $\left(550 \mathrm{~km} \mathrm{~s}^{-1}\right)$ at the coronal base. The broad range of mass ejection speeds can be understood in terms of closed magnetic structures going out of equilibrium to expand into interplanetary space. It is the magnetic tension force of a closed magnetic field that enables the trapping of plasma in helmet-like structures against the general tendency to expand outward with the solar wind. Under continual stressing in the course of gradual coronal evolution, a failure of the magnetic tension force to confine the plasma would result in a dynamical transition to an outflow. Time dependent self-similar solutions of the full hydromagnetic equations in two and three dimensional space show that the outflow can proceed with a near balance of the forces so that the flow can take place with nearly constant Lagrangian speeds of any magnitude in a broad range (Low 1984).

This physical picture of the coronal mass ejection attributes the basic cause to an instability in the large scale coronal magnetic field (Low 1982, Wolfson 1982, Sime 1989). It is supported by the observation that a majority of mass ejections originate from the disruption of pre-existing helmet structures. A helmet structure usually contains a prominence filament at the base, which almost always erupts along with the disruption of the helmet structure (Hundhausen 1987). The 
second point of interest is that the eruption of the prominence often follows after the mass ejection has ascended high in the corona in full motion, precluding the former as a driver of the mass ejection. More dramatic is the case where the mass ejection occurs in association with a solar flare. In this case, the mass ejection is observed almost always to precede the flare by some 20 minutes (Harrison 1986). An early idea that the flare's enormous energy drives the mass ejection is therefore not tenable.

As a finite amplitude motion in the large scale corona, the mass ejections may be expected to be driving hydromagnetic shocks. In the corona, the typical Alfven speed is greater than the sound speed. To drive a fast hydromagnetic shock, the driver must move at a super-Alfvenic speed. The very fast mass ejections probably are capable of driving fast hydromagnetic shocks and a few specific events showing evidence of this have been found (Sime and Hundhausen 1987). But, for the majority of the mass ejections which move at sub-Alfvenic speeds, no associated fast shocks can be expected. Since many of these mass ejections have speeds which are supersonic, it is possible that they generate and drive slow hydromagnetic shocks (Hundhausen, Holzer and Low 1987). Possible evidence of the presence of slow shocks has been found and interest in this and other lesser known types of hydromagnetic shocks has been renewed (Hu et al. 1989, Steinolfson and Hundhausen 1989a, 1989b).

We have made considerable progress in the study of the dynamics of the coronal magnetic field, to the point where phenomenology has touched base with physical principles. Our direct acquaintance with the corona offers a first insight into similar hydromagnetic processes that probably also take place elsewhere under more exotic astrophysical circumstances.

The National Center for Atmospheric Research is sponsored by the US National Science Foundation. The Solar Maximum Mission Satellite Coronagraph/Polarimeter is operated by the High Altitude Observatory under contract with the US National Aeronautic and Space Administration.

\section{References}

Harrison, R. A. 1986, Solar coronal mass ejections and flares, Astron. Astrophys. 162, 283.

Hu, Y. Q., Zhu, Z. W., Hundhausen, A. J., Holzer, T. E., and Low, B. C. 1989, Slow shocks in an open magnetic field near the sun, Scientia Sinica, in press.

Hundhausen, A. J. 1987, The origin and propagation of coronal mass ejections, in Proc. of Sixth International Solar Wind Conference, edited by V. Pizzo, T. Holzer and D. Sime. (NCAR Publ. TN-306).

Hundhausen, A. J., Holzer, T. E., and Low, B. C. 1987, Do slow shocks precede some coronal mass ejections, J. Geophys. Res. 92, 11173.

Kahler, S. 1987, Coronal mass ejections, Rev. Geophys. 25, 663.

Low, B. C. 1982, Eruptive solar magnetic fields, Ap. J. 251, 352.

Low, B. C. 1984, Self-similar magnetohydrodynamics IV. The physics of coronal mass ejections, Ap. J. 281, 392.

Low, B. C. 1986, Coronal mass ejections, in Highlights of Astronomy, edited by J. P. Swing (Reidel). MacQueen, R. M. 1980, Coronal transients: a summary, Phil. Trans. R. Soc. (Lon.) A297, 605.

Sime, D. G. 1989, Coronal mass ejection rates and the evolution of the large scale K-coronal density distribution, J. Geophys. Res. 94, 151.

Sime, D. G., and Hundhausen, A. J. 1987, The coronal mass ejections of July 6 1980: A candidate for interpretation as a coronal shock wave, J. Geophys. Res. 92, 1049. 
Steinolfson, R. S., and Hundhausen, A. J. 1989a, Waves in low-beta plasmas: slow shocks, J. Geophys. Res. 94, 1222.

Steinolfson, R. S., and Hundhausen, A. J. 1989b, MHD intermediate shocks in coronal mass ejections, J. Geophys. Res., in press.

Wolfson, R.L.T. 1982, Equilibria and stability of coronal arches, Ap. J. 255, 774.

PUDRITZ: What determines how much mass is carried out by these erupting structures? Do theoretical calculations agree with the observations?

LOW: A significant majority of coronal mass ejections result from the disruption and outward expansion of pre-existing helmet-like coronal structures. Observation shows that the $10^{15}-10^{16} \mathrm{~g}$ of ionized material in the former can be explained in terms of the mass in the pre-eruption helmet structure. It can be shown theoretically that this amount of mass can be confined in magnetostatic equilibrium by the magnetic field of the helmet structure.

DEINZER: How can you see a prominence if it is taking place below a helmet structure, where the density is increased?

LOW: The prominence embedded in the dense helmet structure is indeed often difficult to detect at the solar limb. The prominence sits in a (low-density) cavity. When a helmet structure at the solar limb happens to be so aligned that we look down the long cavity (along the prominence length), the prominence then shows up as a bright knot in the conspicuous dark cavity.

FEDORENKO: What do you think about the contribution of the solar shock waves to the particle acceleration, viz. cosmic rays?

LOW: Solar physicists believe that a propagating fast shock can accelerate electrons to give rise to Type II radio noise. I should clarify that in my talk I was discussing the evidence of shocks that can be found in coronagraph data. The evidence in the data is expected to be weak. A discontinuity in volume density at a shock shows up as a discontinuity in the spatial gradient of the line-of-sight integral of the density, which is what the coronagraph essentially measures.

HEILES: In regions where the magnetic field strength is large is the gas volume density also large?

LOW: If excess mass is introduced into a magnetic field over scales small compared to that of the field, we would expect the field strength to be small where the density is high, over these small scales. If the added mass and magnetic field vary over comparable scales, high density may correlate with high field strength over the larger scales, with the magnetic tension force providing a means of confinement. One can think of introducing excess mass into the strong field region of an initially, spatially varying, potential field. 